\title{
THE DEVELOPMENT OF LOCOMOTOR ACTIVITY IN DROSOPHILA MELANOGASTER LARVAE
}

\author{
RAUL GODOY-HERRERA,* BARRIE BURNET, KEVIN CONNOLLY AND \\ JOHN GOGARTY \\ Departments of Genetics and Psychology, University of Sheffield, England
}

Received 29.vii.83

\section{SUMMARY}

\begin{abstract}
During the trophic phase of their development Drosophila melanogaster larvae show a preference for moist substrates but mature larvae switch to preference for a dry substrate with the onset of pupation. The larvae also exhibit orthokinesis-an increase in speed of locomotion in the absence of food. The effective range of foraging activity measured in terms of ability to locate and move into a food source improves as development proceeds. Speed of locomotion increases with larval age, largely as a consequence of gain in body size, but also as a consequence of genetically controlled changes in locomotor activity per se. There is substantial directional dominance for increase in locomotor activity with age.
\end{abstract}

\section{INTRODUCTION}

How a behaviour pattern develops during the life history of individuals is one of four fundamental questions for ethology (Tinbergen, 1963). Such changes should at least in part be under genetic control, and it is important therefore to extend investigations beyond the descriptive level to the underlying genetic structure of the behaviour (Manning, 1976; Bateson, 1981). As development proceeds the epigenetic relationship between genes may be modified, similarly the ecological demands on an individual change during its life history. Consequently the nature of the interaction between the genotype involved in the control of a behaviour pattern and the environment may alter at different stages in development (Waddington, 1953). Analyses of the effects of genetic differences on the expression of behaviour should aid our understanding of how behaviour patterns are organised and what contribution they make to fitness.

The larva of $D$. melanogaster shows a variety of behavioural responses closely associated with feeding and with the selection of a suitable pupation site. Green et al., (1983) describe a number of discrete components of behaviour shown by larvae and present a description of the structure of such behaviour based upon transitions between components. On a nonnutritive substrate the predominant behaviour is locomotion accompanied by exploratory movements. These together constitute foraging. When in contact with food the predominant behaviour changes to feeding which involves rhythmic extension and contraction of the mouth hooks. Larvae also show a strong propensity to dig into the substrate (Godoy-Herrera, 1977, 1978). Foraging (Sokolowski, 1980; Sokolowski et al., 1983), feeding activity (Sewell et al., 1975; Burnet et al., 1977), and digging (Godoy-

* Present address: Universidad de Chile, Facultad de Medicina Norte, Departamento de Biologia Celular y Genetica, Casilla 6556, Santiago 7, Chile. 
Herrera, op. cit.) are each individually responsive to selection, and under multigenic control.

Here we focus upon one component of the behaviour of the larva, locomotor activity, and examine this longitudinally at different stages in the life history of the larva and in relation to its genetic organisation.

\section{MATERIALS AND METHODS}

\section{(i) Stocks}

The stocks used for these investigations were: (i) a massbred strain Canton-S; (ii) five isogenic lines designated Leeds M1, M2, M4, M5 and M6, respectively. The Canton-S line was originally an inbred strain of long standing which had been released from close inbreeding some time before these experiments began. The Leeds isogenic lines originated from wildinseminated females (isofemale lines) caught in a fruit and vegetable market and maintained under constant single pair brother sister mating for many generations. A description of the wild population is given by Atkinson and Shorrocks (1977). Stocks were maintained on a standard agar-oatmealmolasses-live yeast medium at $25^{\circ} \mathrm{C}$ with a light-dark cycle of 12 hours light and 12 hours dark. All behavioural observations were made at $25 \pm 1^{\circ} \mathrm{C}$.

\section{(ii) Egg collections and timed material}

Mature 2-3 day old females of the Canton-S strain were allowed to oviposit at low density on plastic spoons filled with culture medium and spread with a thin layer of live baker's yeast for a period of four hours. Fresh live yeast was added daily to minimise larval competition for food. Larval dispersal and locomotor activity in relation to environmental variables were then scored at $24,48,72$ and 96 hours post-hatching as described below.

Measurements of larval body length were made on groups of larvae of known age which were fixed in an extended state by plunging them live into water at $70^{\circ} \mathrm{C}$. The larvae were then surface dried on tissue and their overall length measured.

For the diallel cross, matings were made in all $5 \times 5$ combinations between the five Leeds strains using 2-3 day old virgin flies. After 24 hours, egg collections were made by transferring parents of each genotype group to empty bottles inverted over watch glasses containing medium spread with a thin lawn of live baker's yeast. A two-hour period was allowed for oviposition. The watch glasses were then removed and incubated. Fresh live yeast was added after eclosion of the larvae. Twenty-four hours after hatching from the egg, the locomotor activity of larvae from each cross in the diallel matrix were scored individually. Each larva was then cultured individually on a watch glass (spread with live yeast and renewed each day) until,it reached the age of 72 hours when its locomotor activity was again measured. In this way successive scores at each age were obtained for every larva. Block replication was achieved by making a second egg collection, and taking a further sample of larvae from each of the 25 genotype groups in the diallel cross matrix. These were treated and measured in the same way as the larvae used in block 1 . Some larvae tested at 24 hours died 
before they reached the age of retesting at 72 hours. The data for these larvae were discarded. The analysis was based on four replicate larvae per cell (100 larvae per block).

\section{(iii) Binary and ternary choices}

To test the ability of larvae to locate a food source a $15 \times 15 \times 2 \mathrm{~cm}$ perspex box was filled to a depth of $0.25 \mathrm{~cm}$ with 3 per cent plain agar and spread with a layer of fresh live baker's yeast paste covering a $2 \times 15 \mathrm{~cm}$ strip at one end. Fifty larvae were then placed on the opposite side $13 \mathrm{~cm}$ away from the yeast. The number of larvae on the agar and on the yeast was counted at successive $10 \mathrm{~min}$ intervals over a total observation period of $60 \mathrm{~min}$. Each test was replicated four times with each larval age group.

Larval preferences in respect of moist and dry conditions were tested by filling a $15 \times 15 \mathrm{~cm}$ box with agar as above and then removing a $15 \times 7 \mathrm{~cm}$ area of agar to leave a dry surface. Fifty larvae were then placed on the agar $7 \mathrm{~cm}$ from the division between the moist agar and the dry surface. The number of larvae on each surface was counted every 10 min over a 60 min observation period. Each test was replicated four times with each age group.

The behaviour of larvae presented with a ternary choice between dry, moist and a food containing substrate was achieved by filling a $15 \times 15 \mathrm{~cm}$ box with a $0.25 \mathrm{~cm}$ layer of agar, then cutting away the perimeter to leave a $6 \times 6 \mathrm{~cm}$ area of agar surrounded by a dry surface. A $6 \times 1 \mathrm{~cm}$ strip of live yeast paste was then layered onto one side of the agar. Fifty larvae were placed on the agar along the opposite margin $5 \mathrm{~cm}$ from the yeast. The numbers of larvae on the dry surface, the agar and the yeast were then counted every 10 min over a one hour period. Four replicate trials for each group were used.

\section{(iv) Larval dispersal}

Larval dispersal was measured by placing a group of 20 larvae on agar in a small area at the centre of a $15 \times 15 \times 2 \mathrm{~cm}$ perspex box filled to a depth of $0.5 \mathrm{~cm}$ with 3 per cent agar. The relative position of each larva was recorded at successive $30 \mathrm{sec}$ intervals over a total sample period of $180 \mathrm{sec}$. Larval distributions were analysed using the nearest neighbour method of Clark and Evans (1954). The average distance to neighbour $\bar{r}_{A}$ was compared with the value expected $\bar{r}_{E}$ for the same number of individuals randomly distributed in an area of equal size. $\tilde{r}_{E}=1 / 2 \sqrt{\rho}$, where $\rho$ is the larval density. The ratio $R=\bar{r}_{A} / \bar{r}_{E}$ reflects the form of the spatial distribution of individuals (aggregated, random, overdisperse). Its value varies between limits of $R=0$ (maximum aggregation) and $R=2 \cdot 15$ (uniform). When individuals are randomly distributed then $R=1$.

\section{(v) Locomotor activity}

Locomotor activity was examined using shallow rectangular perspex boxes $15 \times 15 \times 2 \mathrm{~cm}$, filled to a depth of $0.5 \mathrm{~cm}$ with 3 per cent agar and covered by a transparent lid on the outer surface of which was etched a grid of $1 \mathrm{~cm}$ squares. Larvae of the Canton-S strain were transferred 
individually to the agar surface and the number of squares entered was scored in each successive $30 \mathrm{sec}$ interval for a total observation period of $180 \mathrm{sec}$. Larvae of the Canton-S strain were scored at 24, 48, 72 and 96 hours post-hatching age. Fifty larvae from each age group were scored.

For the diallel analysis of locomotor activity the procedure differed from the above in that a finer grid of $0.5 \mathrm{~cm}$ squares was used giving a more sensitive measure. Also, each larva was given a settling down period of 1 min following transfer to the observation box. Activity was then scored over the succeeding $60 \mathrm{sec}$ period.

\section{Results}

\section{(i) Larval substrate preferences}

The substrate preferences exhibited by larvae of different ages were examined initially in a binary choice situation. Larvae were first given a choice between a moist agar substrate and a dry surface. The larvae were placed on the agar adjacent to the dry surface. Larvae aged 24,48 and 72 hours remained on the moist agar, moving over the surface, and returned onto it when they encountered the junction with the dry area. The behaviour of late third instar (96 hours) larvae differed from that of the younger larvae in that when they encountered the dry substrate they tended to move onto it, as shown in fig. 1(a).
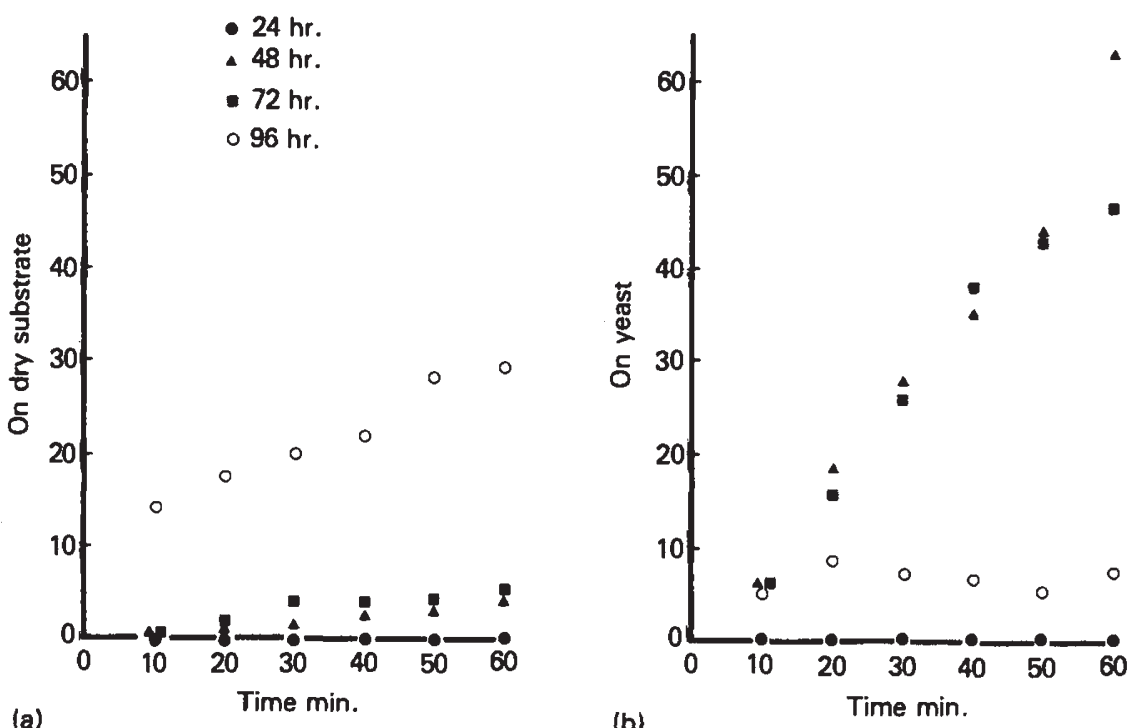

FIG. 1. Binary choice tests for larval substrate preferences. (a) Percentage of larvae leaving agar and moving onto the dry substrate during a $60 \mathrm{~min}$ observation period. (b) Percentage of larvae leaving agar and moving into yeast during a 60 min observation period. Larvae were aged $24,48,72$ and 96 hours post-hatch.

The relative ability of larvae of different ages to successfully locate a food source at a distance, and then enter and exploit it, is illustrated in fig. 1(b). The larvae were placed on agar along a line $13 \mathrm{~cm}$ away from an area of live yeast paste and then allowed to crawl freely over the agar. The 48 
and 72 hours old larvae tended to move into the yeasted area and remain there feeding. The 96 hours old larvae tended to stay on the agar and those entering the yeasted area, unlike the younger individuals, moved out again. Although crawling over the moist agar surface, none of the 24 hours old larvae had entered the yeasted area by the end of the 60 min observation period.

The differences in substrate preference of larvae of different ages exhibited in the binary choice trials were re-examined in a ternary choice situation in which all three types of substrate were presented. Larvae were placed on the moist agar surface adjacent to the margin with the dry surface and along a line now only $5 \mathrm{~cm}$ from the yeasted area. The results are illustrated in fig. 2 .

The 48 and 72 hour old larvae again moved progressively into the yeasted area and remained there feeding, so that nearly all larvae of these two age groups were on the yeast within 1 hour from the start of the trial. The 24 hour old larvae remained predominantly on the agar surface, a small proportion (14 per cent) reaching and entering the yeast and some ( 6 per cent) moved out on to the dry surface. The mature 96 hour old larvae moved progressively off the agar and onto the dry surface. The majority (61 per cent) were located there by the end of the observation period.

These results indicate that larvae prefer different substrates at different points in their development. The 48 and 72 hour old larvae avoid dry, and prefer a moist substrate over which they forage. On locating an available food source they enter it and begin to exploit it. In contrast mature late third instar larvae show a distinct preference for a dry substrate. This correlated with the reduction and eventual cessation of feeding activity in late third instar larvae which, at the onset of pupariation, develop a preference for dryer pupation sites out of the food medium (see review by Grossfield, 1978).

First instar larvae are active and begin to feed shortly after hatching from the egg. They move preferentially into yeast to begin feeding when the food source is close to them (unpublished observations). Consequently, what the results of the binary and ternary choice trials show is that the effective range of foraging activity in 24 hour old larvae is smaller than that of 48 and 72 hour old larvae, not that their substrate preference is different. An apparently narrow range of effective foraging activity in younger larvae could depend upon the distance over which they can detect olfactory cues indicating the presence of food, and upon their locomotor activity.

\section{(ii) Larval dispersal in and out of food}

When a number of larvae are placed in very close proximity in a small area at the centre of a uniform substrate they tend to disperse so that the average distance between neighbouring individuals increases with time. Nearest neighbour analysis yields a ratio $R$ which reflects the pattern of spatial dispersion of larvae. The rate of change of $R$ is also, under limited conditions, a measure of the locomotor activity of the larvae. Fig. 3 shows a comparison of the dispersal of groups of larvae of different ages, on a uniform surface of medium without yeast, and also on a lawn of fresh live yeast. The larvae are initially aggregated $(R<1)$, but in each age group 

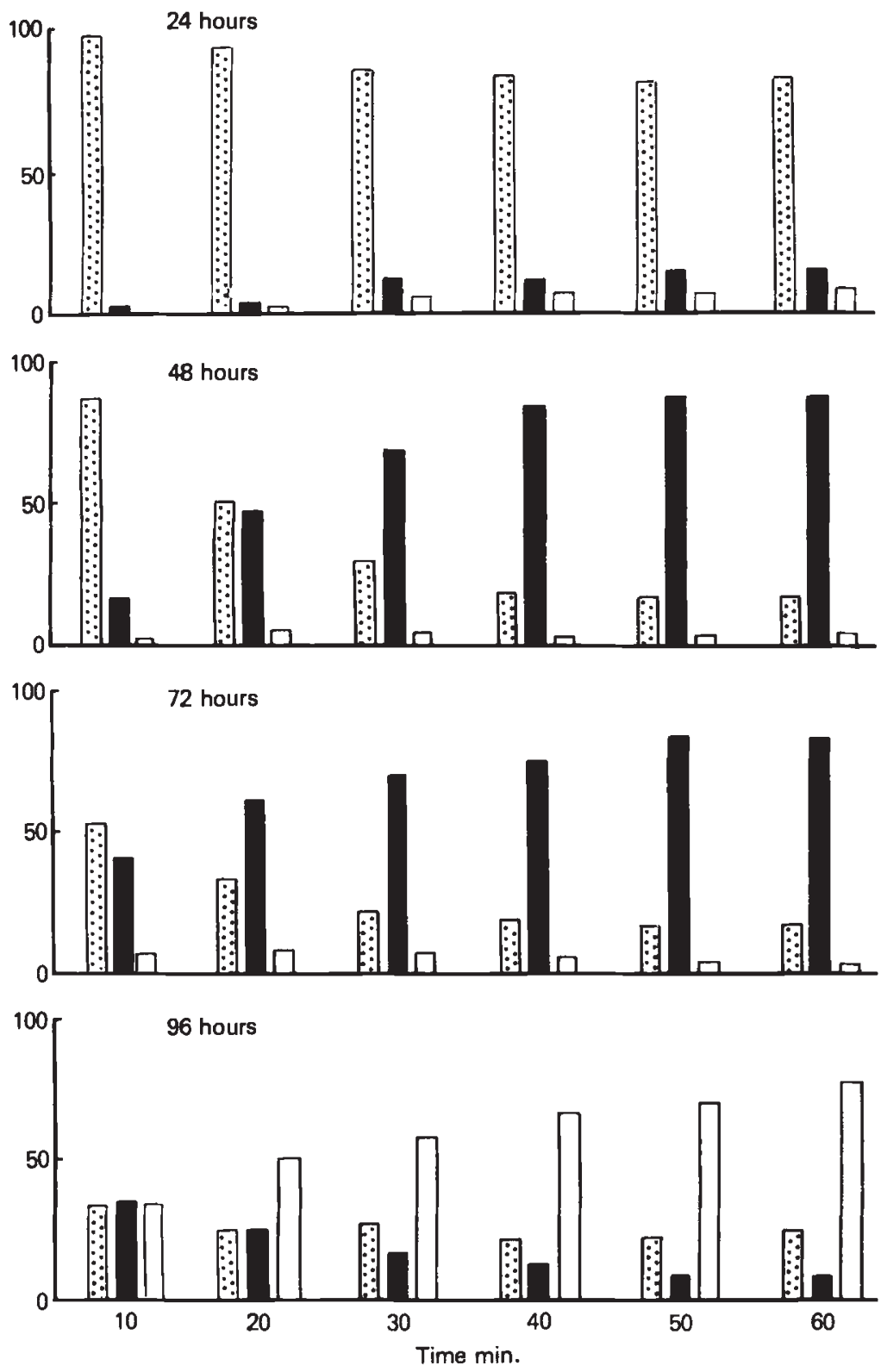

Agar Yeast $\square$ Dry substrate

FIG. 2. Percentage of larvae of different ages on agar, yeast, and a dry substrate after successive $10 \mathrm{~min}$ intervals in the ternary choice test. Larvae were placed initially on the agar.

the distribution tends towards randomness $(R=1)$ with time. Larvae of the same age group show a significant difference in the rate of increase in $R$ in the two situations at 24,48 and 72 hours (table 1 ), which indicates that there are differences in locomotor activity depending on whether they are in contact with food. Larvae on the non-nutritive substrate show a faster 
TABLE 1

Regression coefficients for the change in $R$ over age, for larvae in the presence and absence of food. $p<0.01$ indicates a difference between regression coefficients significant at the 1 per cent level of probability

\begin{tabular}{cccc}
\hline Age & without yeast & with yeast & $p$ \\
\hline $24 \mathrm{hr}$ & $0 \cdot 00322 \pm 0 \cdot 00138$ & $0 \cdot 00102 \pm 0 \cdot 00064$ & $<0.01$ \\
$48 \mathrm{hr}$ & $0 \cdot 00438 \pm 0 \cdot 00114$ & $0 \cdot 00189 \pm 0 \cdot 00057$ & $<0 \cdot 01$ \\
$72 \mathrm{hr}$ & $0 \cdot 00480 \pm 0 \cdot 00059$ & $0 \cdot 00319 \pm 0 \cdot 00074$ & $<0 \cdot 01$ \\
$96 \mathrm{hr}$ & $0 \cdot 00203 \pm 0 \cdot 00322$ & $0 \cdot 00111 \pm 0 \cdot 00168$ & n.s. \\
\hline
\end{tabular}

rate of dispersal than larvae of the same age group in yeast. This would be predicted from the results reported by Green et al., (1983), who found that larvae on non-nutritive substrates showed higher levels of locomotor activity and exploratory behaviour. Lower levels of locomotor activity were associated with feeding activity on yeast.

The results shown in fig. 3 and table 2 suggest that the late third instar larvae (96 hours) do not show a significant difference in their rates of

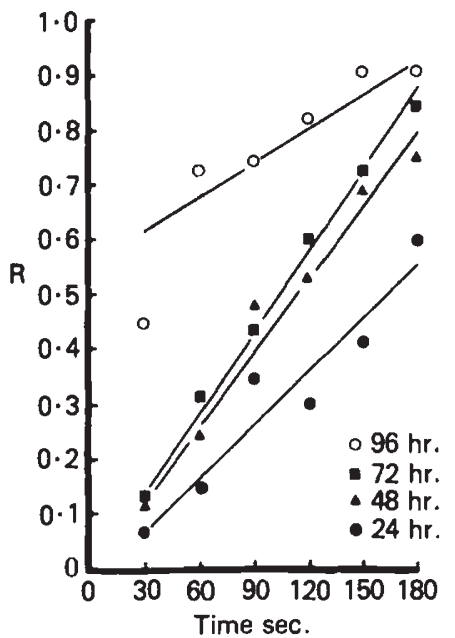

(a)

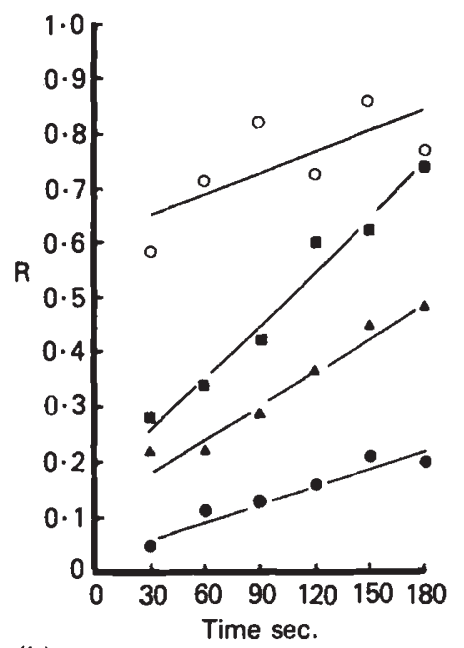

(b)

F1G. 3. The rates of dispersal of larvae in different age groups shown as increase in $R$ (ordinate) with time; (a) in the absence of yeast, (b) on yeast. Canton-S strain.

TABLE 2

Mean difference in locomotor activity in larvae between 24 and $72 \mathrm{hr}$ of age for the Leeds strains and their reciprocal $F_{1}$ hybrids in the diallel cross

\begin{tabular}{cccccc}
\hline & \multicolumn{5}{c}{ Female parents } \\
\cline { 2 - 6 } Male \\
\cline { 2 - 6 } & M1 & M3 & M4 & M5 & M6 \\
\hline M1 & 1.25 & 1.75 & 1.25 & 3.13 & 1.38 \\
M3 & 1.63 & 0.50 & 1.25 & 1.75 & 2.63 \\
M4 & 2.25 & 2.88 & 0.00 & 1.88 & 0.50 \\
M5 & 1.25 & 2.13 & 3.13 & 1.00 & 1.50 \\
M6 & 0.00 & 1.50 & 1.00 & 1.13 & 1.38 \\
\hline
\end{tabular}


dispersal between nutritive and non-nutritive environments. The availability of food would be expected to diminish in importance as an environmental factor influencing locomotor activity as feeding behaviour attenuates and ceases, with the onset of purpariation.

\section{(iii) Locomotor activity in relation to age}

Comparisons of the effective range of foraging activity of larvae of different ages can be made from observations on individual larvae on non-nutritive agar medium. Fig. 4(a) shows the mean cumulative number of $1 \mathrm{~cm}$ squares entered by each individual, which is an approximate measure of the distance travelled after each time interval. The mean score increases with age from 24 through to 72 hours. Late third instar larvae (96 hours) have lower scores than mid third instar larvae ( 72 hours). For all age groups the increase in score is linear with time after $30 \mathrm{sec}$, indicating that speed of movement is constant. The speed of movement is lower in the first $30 \mathrm{sec}$ due to reactivity to handling. If touched when moving they tend to "freeze" for a short period. Larvae often remain motionless for a few seconds after transfer onto the non-nutritive medium before beginning to locomote, this is believed to reflect reactivity to tactile stimuli in the transfer process.

The effective range of foraging activity evidently increases with age. There are two possible, though not mutually exclusive, explanations for

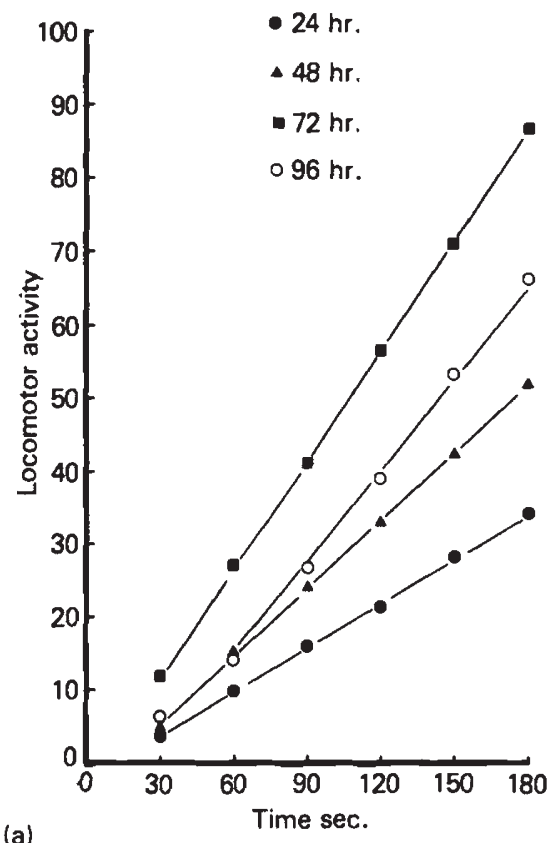

(a)

FIG. 4. (a) Locomotor activity of larvae aged 24, 48, 72 and 96 hours respectively, on agar, shown as the mean number of $1 \mathrm{~cm}$ squares entered after successive $30 \mathrm{sec}$ time intervals. In (b) values on the ordinate have been adjusted to allow for differences in mean body length between the age groups as explained in the text. 
this: (i) the locomotor activity of larvae increases with age; (ii) locomotor activity is constant but, for a given number of locomotor movements, the distance travelled by a big larva is greater than that for a small one. The average length of a 24 hour old larva is $1.55 \mathrm{~mm}$ compared to a mean of $4.70 \mathrm{~mm}$ at 72 hour.

In fig. 4(b) the scores for larvae of different ages have been adjusted using the ratios of the mean body lengths of larvae at 24 hour with each succeeding age group. The effect of this is to show differences in locomotor activity independently of differences in body length. After adjusting for body size, differences in locomotor activity at different stages of development are smaller than fig. 4(a) would suggest. The activity score is highest in the 24 hour old larvae remaining relatively constant between 48 and 72 hours and lowest in the late third instar ( 96 hour). Evidently the increased range of foraging activity exhibited by older larvae is mostly attributable to their larger body size rather than to epigenetically controlled change in their speed of locomotion.

\section{(iv) Genetic control of locomotor activity}

The form of the age related change in locomotor activity in the Canton-S strain, shown above, differs from that described by Burnet and Connolly (1974). In the Florida and Amherst strains locomotor activity, like the feeding movements of the mouth hooks, increased as development proceeded from first to second, and through to early third instar. The difference between these strains and Canton-S suggests that change in the level of expression of larval locomotor activity with age is under genetic control. This was investigated by performing a replicated diallel analysis using the five inbred Leeds melanogaster strains, described above.

Analysis of the results for 72 hour old larvae showed that there were significant differences in locomotor activity between strains, and a Hayman analysis (Hayman 1954a and $b$ ) revealed a low level of additive genetic variance and substantial directional dominance for high activity.

The mean change in locomotor activity score within larvae between 24 and 72 hours post-hatch (adjusted for differences in mean body size) using the five Leeds inbred lines are shown in table 2 together with the corresponding values for the reciprocal crosses between strains. One strain, Leeds M4, showed no age related difference in mean activity score, whereas in each of the other strains locomotor activity increased between 24 and 72 hours post-hatch age which accords with the earlier findings for the Amherst and Florida strains.

The mean for all five inbred parental strains of 0.83 units compares with a mean for the $F_{1}$ reciprocal cross progeny of 1.69 units, yielding a mean potence of 0.87 .

A test for the adequacy of the additive-dominance model, examining the constancy of $\left(W_{r}-V_{r}\right)$ over arrays, is shown in table 3 and indicates an adequate fit with no evidence of epistasis.

The Hayman analysis of variance for data pooled over two blocks is shown in table 4. Tests for significance in the table have been made comparing each item vs. its own interaction with blocks (except for $b 1$ vs. $B T)$. The analysis shows a low additive component of variance which is below statistical significance. There are significant residual dominance 
TABLE 3

Test of the additive dominance model for change in activity $\left(W_{r}-V_{r}\right)$

\begin{tabular}{lcccc}
\hline & $\begin{array}{c}\text { Degrees of } \\
\text { freedom }\end{array}$ & Mean square & $F$ & $P$ \\
\hline Array differences & 4 & 25.5 & 2.86 & n.s. \\
Block differences & 5 & 8.9 & & \\
\hline
\end{tabular}

TABLE 4

Hayman's analysis of variance for changes in larval locomotor activity pooled over two blocks. Analysis of cell means

\begin{tabular}{llrrrr}
\hline Item & Aspect of model tested & $\begin{array}{c}\text { Degree of } \\
\text { freedom }\end{array}$ & $\begin{array}{c}\text { Mean } \\
\text { square }\end{array}$ & $F$ & $P$ \\
\hline$a$ & Additive effects & 4 & $0 \cdot 448$ & $1 \cdot 9$ & n.s. \\
$b$ & Overall dominance & 10 & $0 \cdot 907$ & $4 \cdot 6$ & $0 \cdot 05$ \\
$b 1$ & Directional dominance & 1 & $3 \cdot 019$ & $13 \cdot 8$ & $0 \cdot 01$ \\
$b 2$ & Asymmetry of gene distribution & 4 & $0 \cdot 950$ & $3 \cdot 1$ & n.s. \\
$b 3$ & Residual dominance & 5 & $0 \cdot 451$ & $6 \cdot 8$ & 0.05 \\
$C$ & Maternal effects & 4 & $0 \cdot 326$ & $2 \cdot 3$ & n.s. \\
$d$ & Other reciprocal differences & 6 & $0 \cdot 820$ & $2 \cdot 8$ & n.s. \\
Total & & 24 & & & \\
& & & & & \\
Blocks & & 1 & $0 \cdot 160$ & & \\
$B a$ & & 4 & $0 \cdot 240$ & & \\
$B b$ & 10 & $0 \cdot 197$ & & \\
$B b 1$ & & 1 & $0 \cdot 410$ & & \\
$B b 2$ & & 4 & $0 \cdot 308$ & & \\
$B b 3$ & & 5 & $0 \cdot 066$ & & \\
$B c$ & 4 & $0 \cdot 144$ & & \\
$B d$ & & 6 & $0 \cdot 288$ & & \\
$B T$ & & 24 & $0 \cdot 220$ & & \\
\hline
\end{tabular}

effects, and a significant directional dominance effect for an increase in locomotor activity with age.

\section{Discussion}

Hafez $(1950,1953)$ divided the behaviour of the housefly larva (Musca domestica) broadly into responses of the feeding larva and reactions of the prepupating larva. It seems appropriate to recognise in the development of $D$. melanogaster the same general division of larval behaviour patterns associated with the adaptations of the organism to the trophic and succeeding metamorphic phase of its life history. In contrast to the preference for moist substrates shown in earlier states, near the onset of pupariation late third instar larvae show a preference for drier substrates. A switch in programmed preference occurs (Grossfield, 1978). At present the temporal relationships between the switch to preference for a dry substrate, the cessation of feeding activity, and changes in geotactic and possibly also phototactic responses which occur at this stage are not known. 
The preferences for moist substrates, prior to the switch in the late third instar, are appropriate to the feeding larva since moist conditions are associated with active yeast growth and the availability of food. Larval behaviour in this period is predominantly directed towards acquiring food and reaching critical mass (Burnet et al., 1977). Sokolowski (1980) describes two modes of foraging behaviour in $D$. melanogaster larvae recognised as "rover" which traverse large areas, and "sitter" which tend to remain in a comparatively small area. These two phenotypes were found to be present in a natural population and they may constitute a behavioural polymorphism.

The range of foraging activity, measured in terms of the distance traversed, shows a progressive increase as development proceeds. On the evidence presented here this would seem to be largely a function of the gain in body size with larval age although there is also evidence for age related change in the rate of locomotor activity itself. In first instar larvae foraging activity is evidently less effective than at later stages of development. Pruzan and Bush (1977) and Aceves-Pina and Quinn (1979) have demonstrated chemotactic responses of Drosophila larvae to various stimuli including those provided by yeast and by other larvae. It may be that in younger larvae the distance over which they can detect food is more limited, and the speed at which they can reach it is lower.

Green et al., (1983) measured the rates of locomotor activity in larvae in terms of unitary locomotor movements. They observed an increase in the rate of locomotor activity on non-nutritive substrates compared to the rate on food. They also observed that when larvae encountered food containing elevated concentrations of ethanol, which is potentially toxic, there is reduction in feeding rate and an increase in locomotor activity. In both situations the elevated level of locomotor activity may be seen as an adaptive response to unfavourable environmental conditions, and an example of orthokinesis in the sense used by Fraenkel and Gunn (1940). The increased rates of dispersal of larvae on non-nutritive substrates, described here, represent alternative measures of the same phenomenon. The significant genotype-environment interaction for locomotor activity found by Green et al., (1983), which showed that different strains do not respond in the same way to the presence, versus absence, of food points to the existence of genetic variation affecting the expression of orthokinesis in Drosophila.

The results of the present investigation show that the rate of locomotor activity is developmentally related, and tends to increase with age. There is substantial directional dominance for a high rate of locomotor activity, and for increase in activity with age. Since the scale of the analysis is modest in terms of the numbers of replicate individuals per cell, the fact that the additive components of variance for change in activity is below the level of statistical significance, should not be interpreted to mean that additive genetic variation for this character is absent, but rather that it is at a relatively low level.

The genetical architecture of a behavioural trait may indicate the nature of the relationship between expression of the character and fitness (Broadhurst and Jinks, 1974). In the present case the inference would be that fitness lies in the direction of a high rate of locomotor activity on a non-nutritive substrate, together with increase in level of expression as 
development proceeds. The biological significance of this may lie in the fact that Drosophila larvae are scramble type competitors, exploiting what are in the natural state usually transient environmental conditions, at least with respect to food supply. Yeast growth on natural substrates may often be patchy. When food is exhausted at one location a high level of locomotor activity will extend the effective range of foraging to find food at another. As larvae get older their demand for food increases, raising the potential intensity of competition between individuals. It may, consequently, be advantageous to an individual for the level of locomotor activity in the absence of food to increase in later stages of development.

Acknowledgements. Thanks are due to the British Council for a scholarship which supported Raul Godoy-Herrera and to the Universidad de Chile, Santiago, which gave him leave of absence. John Gogarty is in receipt of a research studentship from the SERC.

\section{REFERENCES}

ACEVES-PINA, E. O. AND QUINN, W. G. 1979. Learning in normal and mutant Drosophila larvae. Science, 206, 93-95.

ATKINSON, W. AND SHORROCKS, B. 1977. Breeding site specificity in the domestic species of Drosophila. Oecologia, 29, 223-232.

BATESON, P. 1981. Ontogeny of behaviour. Brit. Med. Bull., 37, 159-164.

BROADHURST, P. L. AND JINKS, J. L. 1974. What genetical architecture can tell us about the natural selection of behavioural traits. In van Abeelen, J. H. F. (ed.) The Genetics of Behaviour, North-Holland Pub. Co., Amsterdam.

BURNET, B. AND CONNOLLY, K. J. 1974. Activity and sexual behaviour in Drosophila melanogaster. In van Abeelen, J. H. F. (ed.) The Genetics of Behaviour, North-Holland Pub. Co., Amsterdam.

BURNET, B., SEWELL, D. AND BOS, M. 1977. Genetic analysis of larval feeding behaviour in Drosophila melanogaster. II. Growth relations and competition between selected lines. Genet. Res., 30, 149-161.

CLARK, P. J. AND EVANS, F. C. 1954. Distance to nearest neighbour as a measure of spatial relationships in populations. Ecology, 35, 445-453.

FRAENKEL, G. S. AND GUNN, D. L. 1940. The Orientation of Animals. Clarendon Press, Oxford. GODOY-HERRERA, R. 1977. Inter- and intrapopulational variation in digging in Drosophila melanogaster larvae. Behav. Genet., 7, 433-439.

GODOY-HERRERA, R. 1978. Selection for digging behavior in Drosophila melanogaster larvae. Behav. Genet., 8, 475-479.

GREEN, C. H., BURNET, B. AND CONNOLLY, K. J. 1983. Organisation and patterns of interand intraspecific variation in the behaviour of Drosophila larvae. Anim. Behav., 31, 282-291.

GROSSFIELD, J. 1978. Non-sexual behavior of Drosophila. In Ashburner, M. and Wright, T. R. F. (eds.) The Genetics and Biology of Drosophila, vol. 2b, Academic Press, London.

HAFEZ, M. 1950. On the behaviour and sensory physiology of the house-fly larva, Musca domestica. Parasitology, 40, 215-236.

HAFEZ, M. 1953. On the behaviour and sensory physiology of the house-fly larva, Musca domestica. II. Prepupating stage. J. Exp. Zool., 124, 199-225.

HAYMAN, B. 1. 1954a. The analysis of variance of diallel tables. Biometrics, 10, 235-244.

HAYMAN, B. I. 1954b. The theory and analysis of diallel crosses. Genetics, 39, 789-809.

MANNING, A. 1976. The place of genetics in the study of behaviour. In Bateson, P. P. G. and Hinde, R. A. (eds.) Growing Points in Ethology, Cambridge University Press, Cambridge. PRUZAN, A. AND BUSH, G. 1977. Genotypic differences in larval olfactory discrimination in two Drosophila melanogaster strains. Behav. Genet., 7, 457-464.

SEWELL, D., BURNET, B. AND CONNOLLY, K. J. 1975. Genetic analysis of larval feeding behaviour in Drosophila melanogaster. Genet. Res., 24, 163-173.

SOKOLOWSKI, M. B. 1980. Foraging strategies of Drosophila melanogaster: A chromosomal analysis. Behav. Genet., 10, 291-302. 
SOKOlOWSKI, M. B., HANSELl, R. I. C. AND ROTIN, D. 1983. Drosophila larval foraging behavior. II. Selection in the sibling species, D. melanogaster and D. simulans. Behav. Genet., 13, 169-177.

TINBERGEN, N. 1963. On the aims and methods of ethology. Z. Tierpsychol, 20, 410-433. WADDington, C. H. 1953. Epigenetics and evolution. Symp. Soc. Exp. Biol., 8, 186-199. 